\title{
Admissible classes of analytic functions associated with generalized Struve functions
}

\author{
Trailokya Panigrahi and Daniel Breaz
}

\begin{abstract}
In the present paper, by considering suitable classes of admissible functions we investigate some strong differential subordination as well as superordination results for analytic functions associated with normalized form of the generalized Struve functions. As a consequence of these results, new strong differential sandwich-type results are obtained.
\end{abstract}

Mathematics Subject Classification (2010): 30C45, 30C80, 33C10.

Keywords: Analytic functions, strong differential subordination, strong differential superordination, Hadamard product, admissible functions, generalized Struve functions.

\section{Introduction and motivations}

Denote by $\mathcal{H}(\mathbb{U})$, the class of functions which are analytic in the open unit disk

$$
\mathbb{U}:=\{z \in \mathbb{C}:|z|<1\} .
$$

For $a \in \mathbb{C}, n \in \mathbb{N}:=\{1,2,3, \cdots\}$, let

$$
\mathcal{H}[a, n]=\left\{f \in \mathcal{H}(\mathbb{U}): f(z)=a+a_{n} z^{n}+a_{n+1} z^{n+1}+\cdots\right\},
$$

with $\mathcal{H}_{0} \equiv \mathcal{H}[0,1]$ and $\mathcal{H} \equiv \mathcal{H}[1,1]$.

Let $\mathcal{A}$ denote the class of all normalized analytic functions in $\mathbb{U}$ of the form:

$$
f(z)=z+\sum_{n=1}^{\infty} a_{n+1} z^{n+1} \quad(z \in \mathbb{U}) .
$$

For $f, g \in \mathcal{A}$, where $f$ given by (1.1) and $g$ is defined by

$$
g(z)=z+\sum_{n=1}^{\infty} b_{n+1} z^{n+1} \quad(z \in \mathbb{U}),
$$


the Hadamard product (or convolution) of $f$ and $g$, denoted by $f * g$ is defined as

$$
(f * g)(z)=z+\sum_{n=1}^{\infty} a_{n+1} b_{n+1} z^{n+1}=(g * f)(z) .
$$

Let $f, F \in \mathcal{H}(\mathbb{U})$. The function $f$ is said to be subordinate to $F$, or equivalently $F$ is said to be superordinate to $f$, if there exists a function $\omega(z)$ analytic in $\mathbb{U}$, with $\omega(0)=0$ and $|\omega(z)|<1$ such that $f(z)=F(w(z))(z \in \mathbb{U})$. In such a case, we write $f(z) \prec F(z)(z \in \mathbb{U})$. Furthermore, if the function $F$ is univalent in $\mathbb{U}$, then $f(z) \prec F(z) \Longleftrightarrow f(0)=F(0)$ and $f(\mathbb{U}) \subset F(U)$ (see [4]).

Now we consider the following second-order linear non-homogeneous differential equation [14, page 341]):

$$
z^{2} w^{\prime \prime}(z)+z w^{\prime}(z)+\left(z^{2}-p^{2}\right) w(z)=\frac{4\left(\frac{z}{2}\right)^{p+1}}{\sqrt{\pi} \Gamma\left(p+\frac{1}{2}\right)},
$$

where $z \in \mathbb{C}$ and $\Gamma$ stands for the Euler's gamma function. The solution of the homogeneous part is the Bessel's function of order $p$, where $p$ is a real or complex number. The particular integral of (1.2) is called the Struve function of order $p$, given by

$$
H_{p}(z)=\sum_{n=0}^{\infty} \frac{(-1)^{n}}{\Gamma\left(n+\frac{3}{2}\right) \Gamma\left(p+n+\frac{3}{2}\right)}\left(\frac{z}{2}\right)^{2 n+p+1} \quad(z \in \mathbb{C}) .
$$

The differential equation

$$
z^{2} w^{\prime \prime}(z)+z w^{\prime}(z)-\left(z^{2}+p^{2}\right) w(z)=\frac{4\left(\frac{z}{2}\right)^{p+1}}{\sqrt{\pi} \Gamma\left(p+\frac{1}{2}\right)}
$$

differs from (1.2) only in the coefficient of $w$. The particular integral of (1.4) is called the modified Struve function of order $p$ and is given by [14, page 353]:

$$
\begin{aligned}
L_{p}(z) & =-i e^{-\frac{i p \pi}{2}} H_{p}(i z) \\
& =\sum_{n=0}^{\infty} \frac{\left(\frac{z}{2}\right)^{2 n+p+1}}{\Gamma\left(n+\frac{3}{2}\right) \Gamma\left(p+n+\frac{3}{2}\right)} \quad(z \in \mathbb{C}) .
\end{aligned}
$$

Further, let us consider the second-order linear non-homogeneous differential equation of the form (see [6]):

$$
z^{2} w^{\prime \prime}(z)+b z w^{\prime}(z)+\left[c z^{2}-p^{2}+(1-b) p\right] w(z)=\frac{4\left(\frac{z}{2}\right)^{p+1}}{\sqrt{(\pi) \Gamma\left(p+\frac{b}{2}\right)}} \quad(b, c, p \in \mathbb{C}) .
$$

Taking $b=c=1$ and $b=1, c=-1$ in equation (1.6), we get (1.2) and (1.4) respectively. Thus, (1.6) is the generalizes (1.2) and (1.4). This permits us to study the Struve and modified Struve function together. The function $w_{p, b, c}(z)$, called the generalized Sturve function of order $p$ is defined to be the particular integral of (1.6). Moreover, the function $w_{b, p, c}(z)$ has the following familiar representation:

$$
w_{p, b, c}(z):=\sum_{n=0}^{\infty} \frac{(-c)^{n}}{\Gamma\left(n+\frac{3}{2}\right) \Gamma\left(p+n+\frac{(b+2)}{2}\right)}\left(\frac{z}{2}\right)^{2 n+p+1} \quad(z \in \mathbb{C}) .
$$


Note that, the series (1.7) is convergent everywhere but generally not univalent in the open unit disk $\mathbb{U}$.

Recently, Raza and Yağmur [12] (see also, [13]) considered the function $\varphi_{p, b, c}(z)$ defined in terms of the generalized Struve function $w_{p, b, c}(z)$ by the transformation

$$
\begin{aligned}
& \left.\varphi_{p, b, c}(z)=2^{p} \sqrt{(\pi}\right) \Gamma\left(p+\frac{b+2}{2}\right) z^{\frac{-p+1}{2}} w_{p, b, c}(\sqrt{z}) \\
& =z+\sum_{n=1}^{\infty} \frac{\left(\frac{-c}{4}\right)^{n}}{\left(\frac{3}{2}\right)_{n}(\nu)_{n}} z^{n+1} \\
& \left(\nu=p+\frac{b+2}{2} \notin \mathbb{Z}_{0}^{-}:=\{0,-1,-2, \cdots\}, b, p, c \in \mathbb{C}\right),
\end{aligned}
$$

where $(\lambda)_{n}$ denotes the Pochhammer (or Appell) symbol defined by

$$
(\lambda)_{n}=\frac{\Gamma(\lambda+n)}{\Gamma(\lambda)}= \begin{cases}1 & (n=0, \lambda \in \mathbb{C} \backslash\{0\}) \\ \lambda(\lambda+1) \cdots(\lambda+n-1) & (n \in \mathbb{N}, \lambda \in \mathbb{C}) .\end{cases}
$$

For convenience of notation, we write $\varphi_{\nu, c}(z)=\varphi_{p, b, c}(z)$. Now, we introduce a new operator $\mathcal{J}_{\nu}^{c}: \mathcal{A} \longrightarrow \mathcal{A}$ which is defined by means of Hadamard product as

$$
\mathcal{J}_{\nu}^{c} f(z)=\varphi_{\nu, c}(z) * f(z)=z+\sum_{n=1}^{\infty} \frac{(-c)^{n}}{4^{n}\left(\frac{3}{2}\right)_{n}(\nu)_{n}} a_{n+1} z^{n+1} \quad(z \in \mathbb{U}) .
$$

It is easy to verify from (1.8) that

$$
z\left(\mathcal{J}_{\nu+1}^{c} f(z)\right)^{\prime}=\nu \mathcal{J}_{\nu}^{c} f(z)-(\nu-1) \mathcal{J}_{\nu+1}^{c} f(z) .
$$

We need the following definitions and lemmas in order to investigate our main results.

Definition 1.1. (see $[7,8])$ Let $H(z, \xi)$ be analytic in $\mathbb{U} \times \overline{\mathbb{U}}$ and let $f(z)$ be analytic and univalent in $\mathbb{U}$. Then the function $H(z, \xi)$ is said to be strongly subordinate to $f(z)$, or $f(z)$ is said to be strongly superordinate to $H(z, \xi)$, written as $H(z, \xi) \prec \prec f(z)$, if for $\xi \in \overline{\mathbb{U}}, H(z, \xi)$ as the function of $z$ is subordinate to $f(z)$. We note that (see $[1,2,11])$

$$
H(z, \xi) \prec \prec f(z) \quad(z \in \mathbb{U}, \xi \in \overline{\mathbb{U}}) \Longleftrightarrow H(0, \xi)=f(0) \text { and } H(\mathbb{U} \times \overline{\mathbb{U}}) \subset f(\mathbb{U}) .
$$

Definition 1.2. (see $[4,11]$ ) Let $\phi: \mathbb{C}^{3} \times \mathbb{U} \times \overline{\mathbb{U}} \longrightarrow \mathbb{C}$ and let $h(z)$ be univalent in $\mathbb{U}$. If $p(z)$ is analytic in $\mathbb{U}$ and satisfies the following (second-order) differential subordination:

$$
\phi\left(p(z), z p^{\prime}(z), z^{2} p^{\prime \prime}(z), z ; \xi\right) \prec \prec h(z) \quad(z \in \mathbb{U} ; \xi \in \overline{\mathbb{U}}),
$$

then $p(z)$ is called a solution of the strong differential subordination. The univalent function $q(z)$ is called a dominant of the solutions of the strong differential subordination or more simply a dominant, if $p(z) \prec q(z)(z \in \mathbb{U})$ for all $p(z)$ satisfying (1.10). A dominant $\tilde{q}(z)$ that satisfies $\tilde{q}(z) \prec q(z)(z \in \mathbb{U})$ for all dominants $q(z)$ of (1.10) is said to be the best dominant.

Recently, Oros [9] introduced the following notion of strong differential superordination as the dual concept of strong differential subordination. 
Definition 1.3. (see $[5,9])$ Let $\varphi: \mathbb{C}^{3} \times \mathbb{U} \times \overline{\mathbb{U}} \longrightarrow \mathbb{C}$ and let $h(z)$ be analytic in $\mathbb{U}$. If $p(z)$ and $\varphi\left(p(z) z p^{\prime}(z), z^{2} p^{\prime \prime}(z) ; z, \xi\right)$ are univalent in $\mathbb{U}$ for $\xi \in \overline{\mathbb{U}}$ and satisfy the following (second-order) strong differential superordination:

$$
h(z) \prec \prec \varphi\left(p(z), z p^{\prime}(z), z^{2} p^{\prime \prime}(z) ; z, \xi\right) \quad(z \in \mathbb{U}, \xi \in \overline{\mathbb{U}}),
$$

then $p(z)$ is called a solution of the strong differential superordination. An analytic function $q(z)$ is called a subordinant of the solution of the strong differential superordination or more simply a subordinant if $q(z) \prec p(z)$ for all $p(z)$ satisfying (1.11). A univalent subordinant $\tilde{q}(z)$ that satisfies $q(z) \prec \tilde{q}(z)$ for all subordinants $q(z)$ of (1.11) is said to be the best subordinant.

Denote by $Q$, the class of functions $q$ that are analytic and injective on $\overline{\mathbb{U}} \backslash E(q)$, where

$$
E(q)=\left\{\zeta \in \partial \mathbb{U}: \lim _{z \longrightarrow \zeta} q(z)=\infty\right\},
$$

and are such that $q^{\prime}(\zeta) \neq 0$ for $\zeta \in \partial \mathbb{U} \backslash E(q)$. Further, let the subclass of $Q$ for which $q(0)=a$ be denoted by $Q(a), Q(0) \equiv Q_{0}$ and $Q(1) \equiv Q_{1}$.

Definition 1.4. (see [11]) Let $\Omega$ be a set in $\mathbb{C}, q(z) \in Q$ and $n \in \mathbb{N}$. The class of admissible functions $\psi_{n}[\Omega, q]$ consists of those functions $\psi: \mathbb{C}^{3} \times \mathbb{U} \times \overline{\mathbb{U}} \longrightarrow \mathbb{C}$ that satisfy the admissiblility condition $\psi(r, s, t ; z, \xi) \notin \Omega$ whenever

$$
r=q(\zeta), s=\alpha \zeta q^{\prime}(\zeta) \text { and } \Re\left(\frac{t}{s}+1\right) \geq \alpha \Re\left\{1+\frac{\zeta q^{\prime \prime}(\zeta)}{q^{\prime}(\zeta)}\right\},
$$

for $z \in \mathbb{U}, \zeta \in \partial \mathbb{U} \backslash E(q), \xi \in \overline{\mathbb{U}} ; \alpha \geq n$. In particular, for $n=1$, we write $\psi_{1}[\Omega, q]$ as $\psi[\Omega, q]$.

Definition 1.5. (see [9]) Let $\Omega$ be a set in $\mathbb{C}$ and $q \in H[a, n]$ with $q^{\prime}(z) \neq 0$. The class of admissible functions $\psi_{n}^{\prime}[\Omega, q]$ consists of those functions

$$
\psi: \mathbb{C}^{3} \times \mathbb{U} \times \overline{\mathbb{U}} \longrightarrow \mathbb{C}
$$

that satisfy the admissibility condition:

$$
\psi(r, s, t ; \zeta, \xi) \in \Omega
$$

whenever

$$
r=q(z), s=\frac{z q^{\prime}(z)}{m} \text { and } \Re\left(\frac{t}{s}+1\right) \leq \frac{1}{m} \Re\left\{1+\frac{z q^{\prime \prime}(z)}{q^{\prime}(z)}\right\},
$$

for $z \in \mathbb{U}, \xi \in \overline{\mathbb{U}}, \zeta \in \partial \mathbb{U}$ and $m \geq n \geq 1$. In particular, for $n=1$, we denote $\psi_{1}^{\prime}[\Omega, q]$ as $\psi^{\prime}[\Omega, q]$.

For the above two classes of admissible functions, the following results have been proved by earlier authors (see, for details $[9,11]$ ).

Lemma 1.6. (see [11]) Let $\psi \in \psi_{n}[\Omega, q]$ with $q(0)=a$. If $p \in H[a, n]$ satisfies

$$
\psi\left(p(z), z p^{\prime}(z), z^{2} p^{\prime \prime}(z) ; z, \xi\right) \in \Omega
$$

then

$$
p(z) \prec q(z)(z \in \mathbb{U})
$$


Lemma 1.7. (see [9]) Let $\psi \in \psi_{n}^{\prime}[\Omega, q]$ with $q(0)=a$. If the analytic function $p(z) \in$ $Q(a)$ and

$$
\psi\left(p(z), z p^{\prime}(z), z^{2} p^{\prime \prime}(z) ; z, \xi\right)
$$

is univalent in $\mathbb{U}$ for $\xi \in \overline{\mathbb{U}}$, then

$$
\Omega \subset\left\{\psi\left(p(z), z p^{\prime}(z), z^{2} p^{\prime \prime}(z) ; z, \xi\right): z \in \mathbb{U}, \xi \in \overline{\mathbb{U}}\right\}
$$

implies the following subordination relationship:

$$
q(z) \prec p(z)(z \in \mathbb{U}) .
$$

Results dealing with the first-order and the second-order strong differential subordination and strong differential superordination for analytic functions in the open unit disk are available in literature. In recent years, several authors obtained many interesting results involving various linear and non-linear operators associated with strong differential subordination and superordination (see $[1,3,8,9,10,11,14]$ ). By making use of the strong differential subordination and superordination results of Oros and Oros [9, 11], under certain classes of admissible functions we investigate some strong differential subordination and strong differential superordination results of analytic functions associated with the operator $\mathcal{J}_{\nu}^{c}$ defined by (1.8). Further, we find sufficient conditions for suitable classes of admissible functions so that

$$
q_{1}(z) \prec \mathcal{J}_{\nu+1}^{c} f(z) \prec q_{2}(z)
$$

holds true for suitable univalent functions $q_{1}$ and $q_{2}$ with $q_{1}(0)=q_{2}(z)=0$.

\section{Subordination results}

We need the following class of admissible functions in order to prove the subordination results associated with the operator $\mathcal{J}_{\nu}^{c}$ defined by (1.8).

Definition 2.1. Let $\Omega$ be a set in $\mathbb{C}, q \in Q_{0} \cap H_{0}, \Re(\nu)>0$. The class of admissible functions $\phi_{\mathcal{J}}[\Omega, q]$ consists of those functions $\phi: \mathbb{C}^{3} \times \mathbb{U} \times \overline{\mathbb{U}} \longrightarrow \mathbb{C}$ that satisfy the admissibility condition:

$$
\phi(u, v, w ; z, \xi) \notin \Omega
$$

whenever

$$
u=q(\zeta), v=\frac{\alpha \zeta q^{\prime}(\zeta)+(\nu-1) q(\zeta)}{\nu}
$$

and

$$
\begin{gathered}
\Re\left\{\frac{\nu(\nu-1) w-(\nu-1)(\nu-2) u}{\nu v-(\nu-1) u}+(3-2 \nu)\right\} \geq \alpha \Re\left(1+\frac{\zeta q^{\prime \prime}(\zeta)}{q^{\prime}(\zeta)}\right), \\
(z \in \mathbb{U} ; \zeta \in \partial \mathbb{U} \backslash E(q), \xi \in \overline{\mathbb{U}}, \alpha \geq 1) .
\end{gathered}
$$

Theorem 2.2. Let $\phi \in \phi_{\mathcal{J}}[\Omega, q]$. If $f \in \mathcal{A}$ satisfies

$$
\left\{\phi\left(\mathcal{J}_{\nu+1}^{c} f(z), \mathcal{J}_{\nu}^{c} f(z), \mathcal{J}_{\nu-1}^{c} f(z)\right): z \in \mathbb{U}, \xi \in \bar{U}\right\} \subset \Omega,
$$

then

$$
\mathcal{J}_{\nu+1}^{c} f(z) \prec q(z) \quad(z \in \mathbb{U}) .
$$


Proof. Define the function $p(z)$ by

$$
p(z)=\mathcal{J}_{\nu+1}^{c} f(z) \quad(z \in \mathbb{U}) .
$$

Clearly $p(z)$ is analytic in $\mathbb{U}$ with $p(0)=0$. Differentiating $(2.2)$ with respect to $z$ and making use of identity (1.9) in the resulting equation, we get

$$
\mathcal{J}_{\nu}^{c} f(z)=\frac{z p^{\prime}(z)+(\nu-1) p(z)}{\nu} .
$$

Further, a simple calculation shows that

$$
\mathcal{J}_{\nu-1}^{c} f(z)=\frac{z^{2} p^{\prime \prime}(z)+2(\nu-1) z p^{\prime}(z)+(\nu-1)(\nu-2) p(z)}{\nu(\nu-1)}
$$

Now, define the transformations from $\mathbb{C}^{3}$ to $\mathbb{C}$ by

$$
u=r, v=\frac{(\nu-1) r+s}{\nu}, w=\frac{(\nu-1)(\nu-2) r+2(\nu-1) s+t}{\nu(\nu-1)} .
$$

Let

$$
\begin{gathered}
\psi(r, s, t ; z, \xi)=\phi(u, v, w ; z, \xi) \\
=\phi\left(r, \frac{(\nu-1) r+s}{\nu}, \frac{(\nu-1)(\nu-2) r+2(\nu-1) s+t}{\nu(\nu-1)} ; z, \xi\right) .
\end{gathered}
$$

If we use equations $(2.2)-(2.4)$, we find from (2.6) that

$$
\psi\left(p(z), z p^{\prime}(z), z^{2} p^{\prime \prime}(z) ; z, \xi\right)=\phi\left(\mathcal{J}_{\nu+1} f(z), \mathcal{J}_{\nu}^{c} f(z), \mathcal{J}_{\nu-1}^{c} ; z, \xi\right) .
$$

Hence (2.1) becomes

$$
\psi\left(p(z), z p^{\prime}(z), z^{2} p^{\prime \prime}(z) ; z, \xi\right) \in \Omega .
$$

The proof is completed if it can be shown that the admissibility condition for $\phi \in$ $\phi_{\mathcal{J}}[\Omega, q]$ in Definition 2.1 is equivalent to the the admissibility condition for $\psi$ as given in Definition 1.4.

From (2.5), it follows that

$$
\frac{t}{s}+1=\frac{\nu(\nu-1) w-(\nu-1)(\nu-2) u}{\nu v-(\nu-1) u}+(3-2 \nu),
$$

and hence $\psi \in \psi[\Omega, q]$. By Lemma 1.6 we have

$$
p(z) \prec q(z) \quad(z \in \mathbb{U}),
$$

or, equivalently,

$$
\mathcal{J}_{\nu+1}^{c} f(z) \prec q(z) \quad(z \in \mathbb{U}) .
$$

Thus, the proof of Theorem 2.2 is completed.

Corollary 2.3. The conclusion of Theorem 2.2 can be written in the generalized form as:

$$
\left\{\phi\left(\mathcal{J}_{\nu+1}^{c} f(z), \mathcal{J}_{\nu}^{c} f(z), \mathcal{J}_{\nu-1}^{c} f(z) ; \omega(z), \xi\right)\right\} \subset \Omega
$$

then

$$
\mathcal{J}_{\nu+1}^{c} f(z) \prec q(z) \quad(z \in \mathbb{U}),
$$

where $\omega(z)$ is any mapping from $\mathbb{U}$ onto $\mathbb{U}$. 
If $\Omega \neq \mathbb{C}$ is a simply connected domain and $\Omega=h(\mathbb{U})$ for some conformal mapping $h$ of $\mathbb{U}$ onto $\Omega$, then the class $\phi_{\mathcal{J}}[h(\mathbb{U}, q)]$ is written as $\phi_{\mathcal{J}}[h, q]$.

The following result is an immediate consequence of Theorem 2.2.

Theorem 2.4. Let $\phi \in \phi_{\mathcal{J}}[h, q]$. If $f \in \mathcal{A}$ satisfies

$$
\phi\left(\mathcal{J}_{\nu+1}^{c} f(z), \mathcal{J}_{\nu}^{c} f(z), \mathcal{J}_{\nu-1}^{c} f(z) ; z, \xi\right) \prec \prec h(z) \quad(z \in \mathbb{U}, \xi \in \overline{\mathbb{U}}),
$$

then

$$
\mathcal{J}_{\nu+1} f(z) \prec q(z) \quad(z \in \mathbb{U}) .
$$

The following result is an extension of Theorem 2.2 where the behaviour of $q$ on $\partial \mathbb{U}$ is not known.

Corollary 2.5. Let $\Omega \subset \mathbb{C}$ and $q$ be univalent in $\mathbb{U}$ with $q(0)=0$. Let $\phi \in \phi_{\mathcal{J}}\left[\Omega, q_{\rho}\right]$ for some $\rho \in(0,1)$ where $q_{\rho}(z)=q(\rho z)$. If $f \in \mathcal{A}$ satisfies

$$
\phi\left(\mathcal{J}_{\nu+1}^{c} f(z), \mathcal{J}_{\nu}^{c} f(z), \mathcal{J}_{\nu-1}^{c} f(z), z, \xi\right) \in \Omega,
$$

the

$$
\mathcal{J}_{\nu+1} f(z) \prec q(z) \quad(z \in \mathbb{U}) .
$$

Proof. From Theorem 2.2, it follows that

$$
\mathcal{J}_{\nu+1}^{c} f(z) \prec q_{\rho} f(z) .
$$

Since $q_{\rho}(z) \prec q(z)$, hence the result follows.

Theorem 2.6. Let $h$ and $q$ be univalent in $\mathbb{U}$ with $q(0)=0$. Set $q_{\rho}(z)=q(\rho z)$ and $h_{\rho}(z)=h(\rho z)$. Let $\phi: \mathbb{C}^{3} \times \mathbb{U} \times \overline{\mathbb{U}} \longrightarrow \mathbb{C}$ satisfies one of the following conditions:

(i) $\phi \in \phi_{\mathcal{J}}\left[h, q_{\rho}\right]$ for some $\rho \in(0,1)$ or

(ii) there exists $\rho_{0} \in(0,1)$ such that $\phi \in \phi_{\mathcal{J}}\left[h_{\rho}, q_{\rho}\right]$ for all $\rho \in\left(\rho_{0}, 1\right)$.

If $f \in \mathcal{A}$ satisfies (2.7), then

$$
\mathcal{J}_{\nu+1}^{c} f(z) \prec q(z) \quad(z \in \mathbb{U}) .
$$

Proof. Case(i). By applying Theorem 2.2, we obtain $p(z) \prec q_{\rho}(z)$. Since $q_{\rho} \prec q$, we have $p(z) \prec q(z)$.i.e

Case(ii). If we let $p_{\rho}(z)=p(\rho z)$, then

$$
\mathcal{J}_{\nu+1}^{c} f(z) \prec q(z) \quad(z \in \mathbb{U}) .
$$

$$
\begin{gathered}
\phi\left(\mathcal{J}_{\nu+1}^{c} f(z), \mathcal{J}_{\nu}^{c} f(z), \mathcal{J}_{\nu-1}^{c} f(z) ; z, \xi\right) \\
=\phi\left(p_{\rho}(z), \frac{(\nu-1) p_{\rho}(z)+z p_{\rho}^{\prime}(z)}{\nu}, \frac{z^{2} p_{\rho}^{\prime \prime}(z)+2(\nu-1) z p_{\rho}^{\prime}(z)+(\nu-1)(\nu-2) p_{\rho}(z)}{\nu(\nu-1)} ; z, \xi\right) \\
=\phi\left(p(\rho z), \frac{(\nu-1) p(\rho z)+z p^{\prime}(\rho z)}{\nu}, \frac{z^{2} p^{\prime \prime}(\rho z)+2(\nu-1) z p^{\prime}(\rho z)+(\nu-1)(\nu-2) p(\rho z)}{\nu(\nu-1)} ; \rho z, \xi\right) \in h_{\rho}(\mathbb{U}) .
\end{gathered}
$$

By making use of Corollary 2.3 with $\omega(z)=\rho z$, we obtain $p_{\rho}(z) \prec q_{\rho}(z)$ for $\rho \in\left(\rho_{0}, 1\right)$. By letting $\rho \longrightarrow 1$ we obtain

$$
p(z) \prec q(z)
$$

i.e.

$$
\mathcal{J}_{\nu+1}^{c} f(z) \prec q(z) \quad(z \in \mathbb{U}) .
$$

Next theorem gives the best dominant of the strong differential subordination (2.7). 
Theorem 2.7. Let $h(z)$ be univalent in $\mathbb{U}$ and $\phi: \mathbb{C}^{3} \times \mathbb{U} \times \overline{\mathbb{U}} \longrightarrow \mathbb{C}$. Suppose that the differential equation

$$
\phi\left(q(z), \frac{z q^{\prime}(z)+(\nu-1) q(z)}{\nu}, \frac{z^{2} q^{\prime \prime}(z)+2(\nu-1) z q^{\prime}(z)+(\nu-1)(\nu-2) q(z)}{\nu(\nu-1)} ; z, \xi\right)=h(z)
$$

has a solution $q(z)$ with $q(0)=0$ and satisfies any one of the following conditions:

(i) $q \in Q_{0}$ and $\phi \in \phi_{\mathcal{J}}[h, q]$.

(ii) $q$ is univalent in $\mathbb{U}$ and $\phi \in \phi_{\mathcal{J}}\left[h, q_{\rho}\right]$ for some $\rho \in(0,1)$, or

(iii) $q$ is univalent in $\mathbb{U}$ and there exists $\rho_{0} \in(0,1)$ such that $\phi \in \phi_{\mathcal{J}}\left[h_{\rho}, q_{\rho}\right]$ for all $\rho \in\left(\rho_{0}, 1\right)$.

If $f \in \mathcal{A}$ satisfies (2.7) and $\phi\left(\mathcal{J}_{\nu+1}^{c} f(z), \mathcal{J}_{\nu}^{c} f(z), \mathcal{J}_{\nu-1}^{c} f(z) ; z, \xi\right)$ is analytic in $\mathbb{U}$, then

$$
\mathcal{J}_{\nu+1}^{c} f(z) \prec q(z) \quad(z \in \mathbb{U}),
$$

and $q$ is the best dominant.

Proof. By applying Theorem 2.4 and Theorem 2.6 we deduce that $q$ is dominant of (2.7). Since $q$ satisfies (2.8), it is also a solution of (2.7) and therefore $q$ will be the dominant of all dominants of (2.7). Hence $q$ will be the best dominant of (2.7).

In the particular case when $q(z)=M z(M>0)$ and in view of Definition 2.1, the class of admissible functions $\phi_{\mathcal{J}}[\Omega, q]$ denoted by $\phi_{\mathcal{J}}[\Omega, M]$ is described below.

Definition 2.8. Let $\Omega$ be a set in $\mathbb{C}, \nu \in \mathbb{C}$ with $\nu \neq 0,1$ and $M>0$. The class of admissible functions $\phi_{\mathcal{J}}[\Omega, M]$ consists of those functions $\phi: \mathbb{C}^{3} \times \mathbb{U} \times \overline{\mathbb{U}} \longrightarrow \mathbb{C}$ such that

$$
\phi\left(M e^{i \theta}, \frac{\alpha+\nu-1}{\nu} M e^{i \theta}, \frac{L+[2(\nu-1) \alpha+(\nu-1)(\nu-2)] M e^{i \theta}}{\nu(\nu-1)} ; z, \xi\right) \notin \Omega,
$$

$\left(z \in \mathbb{U}, \xi \in \overline{\mathbb{U}}, \Re\left(L e^{-i \theta}\right) \geq \alpha(\alpha-1) M, \theta \in \mathbb{R}, \alpha \geq 1\right)$.

Corollary 2.9. Let $\phi \in \phi_{\mathcal{J}}[\Omega, M]$. If $f \in \mathbb{A}$ satisfies

$$
\phi\left(\mathcal{J}_{\nu+1}^{c} f(z), \mathcal{J}_{\nu}^{c} f(z), \mathcal{J}_{\nu-1} f(z) ; z, \xi\right) \in \Omega \quad(z \in \mathbb{U}, \xi \in \overline{\mathbb{U}}),
$$

then

$$
\mathcal{J}_{\nu+1}^{c} f(z) \prec M z \quad(z \in \mathbb{U}) .
$$

In the special case $\Omega=q(\mathbb{U})=\{w:|w|<M\}$, the class $\phi_{\mathcal{J}}[\Omega, M]$ is simply denoted by $\phi_{\mathcal{J}}[M]$.

Corollary 2.10. Let $\phi \in \phi_{\mathcal{J}}[M]$. If $f \in \mathcal{A}$ satisfies

$$
\left|\phi\left(\mathcal{J}_{\nu+1}^{c} f(z), \mathcal{J}_{\nu}^{c} f(z), \mathcal{J}_{\nu-1}^{c} f(z) ; z, \xi\right)\right|<M \quad(z \in \mathbb{U}, \xi \in \overline{\mathbb{U}}),
$$

then

$$
\left|\mathcal{J}_{\nu+1}^{c} f(z)\right|<M \quad(z \in \mathbb{U})
$$




\section{Superordination and sandwich-type results}

In this section, strong differential superordination, the dual problem of strong differential subordination for generalized Struve function defined as (1.8) is investigated. For this purpose, we define the class of admissible functions as follows:

Definition 3.1. Let $\Omega$ be a set in $\mathbb{C}, q \in H[0,1]$ with $q^{\prime}(z) \neq 0, \Re(\nu)>0$. The class of admissible functions $\phi_{\mathcal{J}}^{\prime}[\Omega, q]$ consists of those functions $\phi: \mathbb{C}^{3} \times \mathbb{U} \times \overline{\mathbb{U}} \longrightarrow \mathbb{C}$ that satisfy the admissibility condition

$$
\phi(u, v, w ; \zeta, \xi) \in \Omega,
$$

whenever

$$
u=q(z), v=\frac{z q^{\prime}(z)+m(\nu-1)}{m \nu} q(z),
$$

and

$$
\begin{gathered}
\Re\left[\frac{\nu(\nu-1) w-(\nu-1)(\nu-2) u}{\nu v-(\nu-1) u}+(3-2 \nu)\right] \leq \frac{1}{m} \Re\left(1+\frac{z q^{\prime \prime}(z)}{q^{\prime}(z)}\right) \\
(z \in \mathbb{U}, \xi \in \overline{\mathbb{U}}, \zeta \in \partial \mathbb{U}, m \geq 1) .
\end{gathered}
$$

Theorem 3.2. Let $\phi \in \phi_{\mathcal{J}}^{\prime}[\Omega, q]$. If $f \in \mathcal{A}, \mathcal{J}_{\nu+1}^{c} f(z) \in Q_{0}$ and

$$
\phi\left(\mathcal{J}_{\nu+1}^{c} f(z), \mathcal{J}_{\nu}^{c} f(z), \mathcal{J}_{\nu-1}^{c} f(z) ; z, \xi\right)
$$

is univalent in $\mathbb{U}$, then

$$
\Omega \subset\left\{\phi\left(\mathcal{J}_{\nu+1}^{c} f(z), \mathcal{J}_{\nu}^{c} f(z), \mathcal{J}_{\nu-1}^{c} f(z) ; z, \xi\right): z \in \mathbb{U}, \xi \in \overline{\mathbb{U}}\right\}
$$

implies the following subordination result holds

$$
q(z) \prec \mathcal{J}_{\nu+1}^{c} f(z) \quad(z \in \mathbb{U}) .
$$

Proof. Let

$$
p(z)=\mathcal{J}_{\nu+1}^{c} f(z)
$$

Then, from (2.6) and (3.1) we obtain

$$
\Omega \subset\left\{\psi\left(p(z), z p^{\prime}(z), z^{2} p^{\prime \prime}(z) ; z, \xi\right): z \in \mathbb{U}, \xi \in \overline{\mathbb{U}}\right\} .
$$

Since

$$
\frac{t}{s}+1=\frac{\nu(\nu-1) w-(\nu-1)(\nu-2) u}{\nu v-(\nu-1) u}+(3-2 \nu),
$$

the admissibility condition for $\phi \in \phi_{\mathcal{J}}^{\prime}[\Omega, q]$ in Definition 3.1 is equivalent to the admissibility condition for $\psi$ as given in Definition 1.5. Hence $\psi \in \psi^{\prime}[\Omega, q]$ and by Lemma 1.7,

$$
q(z) \prec p(z) \quad(z \in \mathbb{U})
$$

or equivalently,

$$
q(z) \prec \mathcal{J}_{\nu+1}^{c} f(z) \quad(z \in \mathbb{U}) .
$$

If $\Omega \neq \mathbb{C}$ is a simply connected domain, then $\Omega=h(\mathbb{U})$ for some conformal mapping $h$ of $\mathbb{U}$ onto $\Omega$. In this case, the class $\phi_{\mathcal{J}}^{\prime}[h(\mathbb{U}), q]$ is written as $\phi_{\mathcal{J}}^{\prime}[h, q]$. The following result is the immediate consequence of Theorem 3.2. 
Theorem 3.3. Let $q \in H[0,1], h$ be analytic in $\mathbb{U}$ and $\phi \in \phi_{\mathcal{J}}^{\prime}[h, q]$. If $f \in \mathcal{A}$, $\mathcal{J}_{\nu+1}^{c} f(z) \in Q_{0}$ and

$$
\phi\left(\mathcal{J}_{\nu+1}^{c} f(z), \mathcal{J}_{\nu}^{c} f(z), \mathcal{J}_{\nu-1}^{c} f(z) ; z, \xi\right)
$$

is univalent in $\mathbb{U}$, then

$$
h(z) \prec \prec \phi\left(\mathcal{J}_{\nu+1}^{c} f(z), \mathcal{J}_{\nu}^{c} f(z), \mathcal{J}_{\nu-1}^{c} f(z) ; z, \xi\right) \quad(z \in \mathbb{U}, \xi \in \overline{\mathbb{U}}),
$$

implies

$$
q(z) \prec \mathcal{J}_{\nu+1}^{c} f(z) \quad(z \in \mathbb{U})
$$

Theorem 3.2 and Theorem 3.3 can only be used to obtain subordinants of differential superordination of the form (3.1) or (3.2). The following theorem proves the existence of the best dominant of (3.2) for an appropriate $\phi$.

Theorem 3.4. Let $h$ be analytic in $\mathbb{U}$ and $\phi: \mathbb{C}^{3} \times \mathbb{U} \times \overline{\mathbb{U}} \longrightarrow \mathbb{C}$. Suppose that the differential equation

$$
\phi\left(q(z), \frac{\left.z q^{(} z\right)+(\nu-1) q(z)}{\nu}, \frac{z^{2} q^{\prime \prime}(z)+2(\nu-1) z q^{\prime}(z)+(\nu-1)(\nu-2) q(z)}{\nu(\nu-1)} ; z, \xi\right)=h(z)
$$

has a solution $q \in Q_{0}$. If $\phi \in \phi_{\mathcal{J}}^{\prime}[h, q], f \in \mathcal{A}, \mathcal{J}_{\nu+1}^{c} f(z) \in Q_{0}$ and

$$
\phi\left(\mathcal{J}_{\nu+1}^{c} f(z), \mathcal{J}_{\nu}^{c} f(z), \mathcal{J}_{\nu-1}^{c} f(z) ; z, \xi\right)
$$

is univalent in $\mathbb{U}$, then

$$
h(z) \prec \prec \phi\left(\mathcal{J}_{\nu+1}^{c} f(z), \mathcal{J}_{\nu}^{c} f(z), \mathcal{J}_{\nu-1}^{c} f(z) ; z, \xi\right) \quad(z \in \mathbb{U}, \xi \in \overline{\mathbb{U}}),
$$

implies

$$
q(z) \prec \mathcal{J}_{\nu+1}^{c} f(z) \quad(z \in \mathbb{U}) .
$$

and $q(z)$ is the best subordinant.

Proof. By applying Theorem 3.3, we deduce that $q$ is a dominate of (3.2). Since $q$ satisfies (3.3), it is also a solution of (3.2) and therefore $q$ will be dominated by all dominates of (3.2). Hence $q$ is the best dominates of (3.2).

Combining Theorem 2.4 and Theorem 3.3, we obtain the following sandwich-type theorem.

Theorem 3.5. Let $h_{1}(z)$ and $q_{1}(z)$ be analytic functions in $\mathbb{U}, h_{2}$ be univalent function in $\mathbb{U}, q_{2} \in Q_{0}$ with $q_{1}(0)=q_{2}(0)=0$ and $\phi \in \phi_{\mathcal{J}}\left[h_{2}, q_{2}\right] \cap \phi_{\mathcal{J}}^{\prime}\left[h_{1}, q_{1}\right]$. If $f \in \mathcal{A}$, $\mathcal{J}_{\nu+1}^{c} f(z) \in H[0,1] \cap Q_{0}$ and

$$
\phi\left(\mathcal{J}_{\nu+1}^{c} f(z), \mathcal{J}_{\nu}^{c} f(z), \mathcal{J}_{\nu-1}^{c} f(z) ; z, \xi\right)
$$

is univalent function in $\mathbb{U}$, then

$$
h_{1}(z) \prec \prec \phi\left(\mathcal{J}_{\nu+1}^{c} f(z), \mathcal{J}_{\nu}^{c} f(z), \mathcal{J}_{\nu-1}^{c} f(z) ; z, \xi\right) \prec \prec h_{2}(z),
$$

implies $q_{1}(z) \prec \mathcal{J}_{\nu+1}^{c} f(z) \prec q_{2}(z),(z \in \mathbb{U})$. 


\section{References}

[1] Antonino, J.A., Romaguera, S., Strong differential subordination to Briot-Bouquet differential equations, J. Differential Equations, 114(1994), 101-105.

[2] Antonino, J.A., Strong differential subordination and applications to univalency conditions, J. Korean Math. Soc., 43(2006), 311-322.

[3] Cho, N.E., Kwon, O.S., Srivastava, H.M., Strong differential subordination and superordination for multivalently meromorphic function involving the Liu-Srivastava operatior, Integral Transforms and Spec. Funct., 21(8)(2010), 589-601.

[4] Miller, S.S., Mocanu, P.T., Differential Subordinations: Theory and Applications, Series on Monographs and Textbooks in Pure and Applied Mathematics, No. 225, Marcel Dekker Incorporated, New York and Bessel, 2000.

[5] Miller, S.S., Mocanu, P.T., Subordinants of differential superordinations, Complex Var. Theory Appl., 48(10)(2003), 815-826.

[6] Orhan, H., Yağmur, N., Geometric properties of generalized Struve functions, in International Congress in Honour of Professor Hari M. Srivastava, Bursa, Turkey, August, 2012.

[7] Oros, G.I., First order differential superordinations using the Dziok-Srivastava linear operator, Math. Reports, 12(62)(2001), 37-44.

[8] Oros, G.I., First order strong differential superordinaton, Gen. Math., 15(2007), no. 2-3, $77-87$.

[9] Oros, G.I., Strong differential superordination, Acta Univ. Apulensis Math. Inform., 19(2009), 101-106.

[10] Oros, G.I., Oros, G., First order linear strong differential subordination, Gen. Math., 15(2007), no. 2-3, 98-107.

[11] Oros, G.I., Oros, G., Strong differential subordination, Turk. J. Math., 33(2009), 249-257.

[12] Raza, M., Yağmur, N., Some properties of a class of analytic functions defined by generalized Struve functions, Turk. J. Math., 39(2015), 931-944.

[13] Yağmur, N., Orhan, H., Starlikeness and convexity of generalized Struve functions, Abst. Appl. Anal., (2013), doi:10.1155/2013/954513.

[14] Zhang, S., Jin, J., Computation of Special Functions, A Wiley Interscience Publication, John Wiley and Sons, New York, NY, USA, 1996.

Trailokya Panigrahi

Department of Mathematics

School of Applied Sciences, KIIT University

Bhubaneswar-751024, Orissa, India

e-mail: trailokyap6@gmail.com

Daniel Breaz

Department of Mathematics

University of Alba Iulia, Romania

e-mail: dbreaz@uab.ro 